\title{
Molecular evidence for the natural production of homozygous Cupressus sempervirens L. lines by Cupressus dupreziana seed trees
}

\author{
JLR Nava ${ }^{1}$, A Buonamici ${ }^{2}$, GG Vendramin ${ }^{2}$ and C Pichot ${ }^{1}$ \\ ${ }^{1} I N R A$, Unité d'Ecologie des Forêts Méditerranéennes (UR629), Domaine St Paul, Site Agroparc, Avignon, France and ${ }^{2} I s t i t u t o ~ d i$ \\ Genetica Vegetale, Consiglio Nazionale delle Ricerche, Sesto Fiorentino (Firenze), Italy
}

\begin{abstract}
Paternal apomixis was recently reported in the endangered Mediterranean cypress, Cupressus dupreziana. This species acts as a surrogate mother for the development of all-paternal embryos from pollen grains. C. dupreziana production of Cupressus sempervirens haploid or diploid seedlings from C. sempervirens pollen was also demonstrated. The haploid progeny was derived from the embryogenic development of haploid gametes, but the origin of the diploid progeny remained unknown. To determine the ontogenic origin of the diploid $C$. sempervirens progeny, we analyzed the heterozygozity of 63 diploid all-paternal $C$. sempervirens seedlings using highly variable co-dominant nuclear microsatellite markers. The bi-parental inheritance of the markers was checked in
\end{abstract}

C. sempervirens controlled crosses. A high level of polymorphism was observed among the diploid all-paternal trees. All but three individuals exhibited single-band profiles as expected for homozygotes, which may originate from natural diploidization of a $C$. sempervirens haploid embryo or from the fusion of two male gametes produced by the same $C$. sempervirens microgametophyte. The three heterozygous seedlings must be derived from the fusion of male gametes produced by two different $C$. sempervirens microgametophytes. These findings offer a unique opportunity in conifers to produce homozygous lines, highly valuable for genetic analyses or breeding. Heredity (2010) 104, 185-190; doi:10.1038/hdy.2009.112; published online 26 August 2009

Keywords: androgenesis; dihaploid; surrogate mother; SSR; conifer

\section{Introduction}

Sexual reproduction is the general rule in higher plants and animals (Bell, 1982; Barton and Charlesworth, 1998; West et al., 1999). However, some species exhibit an apomictic or parthenogenetic reproduction in which the offspring derives from only one parent, the mother (Foroughi-Wehr and Wenzel, 1993). According to McKone and Halpern (2003), very rare cases of androgenesis have been observed in animals. So far, Cupressus dupreziana A. Camus (Cupressaceae) is the only plant species where 'paternal apomixis' has been reported (Pichot et al., 2001).

Cupressus dupreziana (or Tassili cypress) is a highly endangered woody species. It is represented by only one natural population in the Tassili N'Ajjer desert, Algeria, and the number of surviving individuals is limited to 233 (Abdoun and Beddiaf, 2002). This species can easily be distinguished from C. sempervirens L. (common cypress or Mediterranean cypress) on the basis of a multi-locus profile obtained using AFLP (Pichot et al., 2008) and nuclear microsatellites (transferred from Sebastiani et al., 2005). The precarious state of the species results from a combination of extreme climatic conditions, long-term human pressure and extremely scarce regeneration. The

Correspondence: Dr C Pichot, INRA URFM, Domaine St Paul, Site Agroparc, 84914 Avignon Cedex 9, France.

E-mail: pichot@avignon.inra.fr

Received 5 March 2009; revised 15 June 2009; accepted 13 July 2009; published online 26 August 2009 species always exhibits a low seed viability rate, which can be related to the abnormalities of its sexual reproductive process. The aberrant meiosis that occurs in both micro and macrospores leads to the development of diploid pollen grains (Pichot and El Maâtaoui, 2000) and diploid-based megagametophytes (Pichot et al., 1998). The embryogenic ability of C. dupreziana pollen was first demonstrated in inter-specific pollination experiments. All progeny produced by controlled pollination of the Mediterranean cypress (Cupressus sempervirens) using $C$. dupreziana pollen were pure $C$. dupreziana seedlings (Pichot et al., 2001). Reciprocally, the ability of C. dupreziana to act as a surrogate mother for the development of all-paternal $C$. sempervirens progeny was more recently demonstrated (Pichot et al., 2008). Indeed two-thirds of the seedlings produced by open pollinated C. dupreziana trees planted in a South Eastern France collection were pure $C$. sempervirens individuals. Most of these seedlings were haploid, which was consistent with the fact that they would derive from haploid $C$. sempervirens pollen grains without fecundation. On the other hand, the remaining seedlings were diploid and their ontogenic origin remained unknown (Pichot et al., 2008).

In this paper we infer the genetic origin of the diploid all-paternal $C$. sempervirens seedlings using codominant microsatellite markers (single sequence repeat (SSR)) for which we verified the nuclear inheritance. In addition to the progeny included in the study by Pichot et al. (2008), new progeny originating from either open or controlled pollinations were analyzed. The observed genetic 
profiles allowed inference of the ontogenic origin of seedlings.

\section{Materials and methods}

\section{Plant material}

To determine their genetic origin, 63 diploid all-paternal C. sempervirens-like seedlings derived from C. dupreziana seeds were analyzed (Table 1). Most progeny (57) came from open-pollinated C. dupreziana seed trees planted in five ex situ collections (South Eastern France) as described by Pichot et al. (2008). In three of these five cypress collections (Avignon, Carpentras and Montpellier), C. dupreziana seed trees were very close to numerous $C$. sempervirens pollen trees (more than 30 ). In Ruscas collection, the only one $C$. dupreziana seed tree was surrounded by more than $50 \mathrm{C}$. sempervirens and 50 Cupressus arizonica pollen trees. The last site (Esterel collection) was a pure $C$. dupreziana collection at a distance of about $2 \mathrm{~km}$ from the closest $C$. sempervirens and C. arizonica trees.

Six progeny produced by controlled pollinations of one C. dupreziana seed tree with pollen collected from three C. sempervirens trees were also analyzed. Seedling ploidy level was assessed by flow-cytometry upon following the protocol described by Pichot and El Maâtaoui (2000).

\section{Molecular marker selection and inheritance}

Seedlings were genotyped using seven nuclear microsatellites (SSRs) developed for C. sempervirens: Cyp52, Cyp84, Cyp101, Cyp174, Cyp257, Cyp258 and Cyp293 (Sebastiani et al., 2005). These markers were highly polymorphic in C. sempervirens populations (Valgimigli, 2005), providing high resolution for paternity analysis and heterozygote detection, but their putative nuclear inheritance had never been verified. As known marker inheritance is a prerequisite for studying the genetic origin of progeny, Mendelian segregation of the SSR markers was tested by analyzing the genotypes of 77 C. sempervirens seedlings from five full-sib families, including two reciprocal crosses, and the six parents of the crosses (see Supplementary information Table 2). This material was selected from a C. sempervirens $9 \times 9$ diallele mating design (unpublished data). Leaf samples were collected from 5-year old individual C. sempervirens planted in a progeny trial near Avignon, South Eastern France.

\section{Genotyping protocol}

Genotyping was carried out in two different laboratories, at CNR-Firenze (Italy) (first set of samples) and at INRAAvignon (France) (second set of samples). The first set of samples included all the seedlings used for the marker inheritance analysis and 31 of the 63 diploid all-paternal individual seedlings were derived from $C$. dupreziana seed trees. The remaining 32 diploid all-paternal individual seedlings were included in the second set of samples. DNA was extracted from leaf samples using the DNeasy plant kit (Qiagen, Venlo, The Netherlands).

For the first set of samples, PCRs were carried out in $25 \mu \mathrm{l}$ containing $10 \mathrm{ng}$ of DNA, $1 \times$ PCR reaction buffer (Promega, Madison, WI, USA), $200 \mu \mathrm{M}$ of each dNTP, 1-U Taq polymerase (GoTaq, Promega), $1.5 \mathrm{mM} \mathrm{MgCl}_{2}$, and $0.2 \mu \mathrm{M}$ of each primer. The forward primers were labeled with FAM, HEX or TAMRA. The PCR thermal profile (except for Cyp258, see Sebastiani et al. (2005)) was as follows: denaturation at $94{ }^{\circ} \mathrm{C}$ for $5 \mathrm{~min}$ followed by 30 cycles of $94{ }^{\circ} \mathrm{C}$ for $30 \mathrm{~s}, 50{ }^{\circ} \mathrm{C}$ for $30 \mathrm{~s}, 72^{\circ} \mathrm{C}$ for $30 \mathrm{~s}$ and a final extension of $72{ }^{\circ} \mathrm{C}$ for $8 \mathrm{~min}$. PCRs were carried out using a Perkin-Elmer GeneAmp 9700 thermal cycler (Applied Biosystems, Foster City, CA, USA). The PCR products were separated by capillary electrophoresis, with a 400 bp size standard, using MEGABACE 1000 (GE Healthcare, Chalfont, St Giles, UK) automatic sequencer. Alleles were sized using FRAGMENT PROFILER version 1.2 (GE Healthcare).

For the second set of samples, three multiplex PCRs (Qiagen), Cyp52 $(700 \mathrm{~nm})+$ Cyp84 $(800 \mathrm{~nm})$, Cyp101 $(700 \mathrm{~nm})+$ Cyp $174 \quad(800 \mathrm{~nm}), \quad$ Cyp257 and Cyp258 $(700 \mathrm{~nm})+$ Cyp $293(800 \mathrm{~nm})$, were carried out in $10 \mu \mathrm{l}$ containing $0.2 \mu \mathrm{g}$ of DNA, $5 \mu \mathrm{l}$ of PCR Master Mix, $1 \mu \mathrm{l}$ of Primer Mix $(0.2 \mu \mathrm{M}$ each primer), $1 \mu \mathrm{l}$ of Q-Solution and $1 \mu \mathrm{l}$ of RNase-free water. Microsatellite loci were analyzed on a LI-COR $\left(4200 \mathrm{IR}^{2}\right)$ automatic sequencer. The size of alleles was determined automatically and (or) manually with the SAGA (LI-COR IR²) computer software.

To check for consistency in allele size estimation across the two laboratories, a common set of PCR products from 19 samples was run on both automatic

Table 1 Origin and genetic diversity of the 63 diploid Cupressus sempervirens progeny produced by 18 Cupressus dupreziana seed trees. The within-site number of single sequence repeat (SSR) alleles and expected heterozygosity (He) are computed from the seedling genotypes and represent the $C$. sempervirens pollen-tree diversity, because of the all-paternal origin of the progeny

\begin{tabular}{|c|c|c|c|c|c|c|c|c|}
\hline \multirow[t]{2}{*}{ Site of collection } & \multirow{2}{*}{$\begin{array}{l}\text { Number of: seed trees } \\
(S T) / \text { progenies }(P)\end{array}$} & \multicolumn{7}{|c|}{ SSR markers diversity (no of alleles $/ H_{e}$ ) } \\
\hline & & cyp52 & cyp84 & cyp101 & cyp174 & cyp 257 & cyp 258 & cyp293 \\
\hline Avignon $^{\mathrm{a}}$ & $1 \mathrm{ST} / 14 \mathrm{P}$ & $2 / 0.13$ & $4 / 0.64$ & $3^{\mathrm{e}} / 0.57$ & $2 / 0.50$ & $3 / 0.56$ & $5 / 0.68$ & $1 / 0$ \\
\hline Carpentras ${ }^{\mathrm{a}}$ & $2 \mathrm{ST} / 10 \mathrm{P}$ & $2 / 0.18$ & $3 / 0.54$ & $2 / 0.32$ & $3 / 0.62$ & $4 / 0.72$ & $3 / 0.34$ & $2 / 0.18$ \\
\hline Estérel $^{\mathrm{b}}$ & $10 \mathrm{ST} / 15 \mathrm{P}$ & $5 / 0.52$ & $6 / 0.71$ & $3^{\mathrm{e}} / 0.42$ & $5 / 0.72$ & $7^{\mathrm{e}} / 0.73$ & $4 / 0.51$ & $4^{\mathrm{e}} / 0.34$ \\
\hline Montpellier ${ }^{c}$ & $4 \mathrm{ST} / 16 \mathrm{P}$ & $6 / 0.63$ & $6 / 0.62$ & $7^{\mathrm{e}} / 0.74$ & $6 / 0.72$ & $4 / 0.67$ & $3 / 0.57$ & $5 / 0.63$ \\
\hline Ruscas ${ }^{b}$ & $1 \mathrm{ST} / 8 \mathrm{P}^{\mathrm{d}}$ & $4 / 0.53$ & $4 / 0.60$ & $2 / 0.49$ & $3 / 0.55$ & $3 / 0.41$ & $2 / 0.12$ & $2 / 0.47$ \\
\hline All sites (weighted by number) & $18 \mathrm{ST} / 63 \mathrm{P}$ & $7 / 0.41$ & $8 / 0.48$ & $7 / 0.53$ & $7 / 0.63$ & $6 / 0.63$ & $8 / 0.48$ & $7 / 0.33$ \\
\hline
\end{tabular}

\footnotetext{
a Private collection.

bINRA ex-situ collections.

${ }^{\mathrm{c}}$ Botanical garden from Montpellier.

${ }^{d}$ Six of the eight progenies were produced by controlled pollination.

'One null allele.
} 
sequencers. These 19 samples, for which a perfect match of the length sizes of the amplified fragments was observed, were used as references in all runs allowing unambiguous comparison of the data obtained in the two laboratories.

\section{Statistical analysis}

Inheritance of markers: The presence of one maternal allele and one paternal allele in all of the C. sempervirens seedlings analyzed made it possible to verify the biparental inheritance of the SSR markers. Moreover allele segregations within full-sib families were analyzed and compared with the expected nuclear Mendelian segregations. The probability of each observed segregation ( $P$-value) was computed from the distribution of the segregations produced by a Monte Carlo procedure (1000 replicates) under the Mendelian inheritance hypothesis. All computations were performed using the $\mathrm{R}$ statistical software (R Development Core Team, 2006).

Probability of single-band SSR profiles: Parent tree SSR genotypes were available for the six progeny produced by controlled pollination. For the other 57 progeny, pollen tree genotypes were unknown and the probability of SSR profiles can, therefore, only be computed from population allele frequencies. Hypothesizing a random mating of gametes, we computed the probability of the single-band profiles that we frequently observed in the diploid all-paternal progeny (see Results section). Computations were carried out using two sets of SSR allele frequencies estimated (a) from the 63 progeny analyzed in the present study and (b) from some Italian cypress populations (Valgimigli, 2005), as an additional control. Indeed, previous results which were based on isozymes demonstrated that French and Italian populations display similar levels of genetic diversity (Dugas, 1996). The probability $P\left(s_{1}\right)$ of a single-band profile at locus $l$ is the probability of two identical alleles (homozygosity) plus the probability of only one null allele:

$$
P\left(s_{l}\right)=\sum_{i=1}^{\# \text { non-null alleles }} P_{i}^{2}+P_{\text {null }} \times\left(1-P_{\text {null }}\right)
$$

where $P_{i}$ is the frequency of the non-null allele $i$ and $P_{\text {null }}$ the frequency of the null allele.

Hypothesizing no linkage disequilibrium among loci, the probability $P\left(S_{71}\right)$ of a single-band profile over the seven loci is:

$$
P\left(s_{71}\right)=\prod_{l o c u s=1}^{7} P\left(s_{l}\right)
$$

Therefore, the probability $P\left(s_{71}^{63} n\right)$ of observing $\mathrm{n}$ singleband individuals among the 63 studied seedlings is:

$$
P\left(s_{71 \_n}^{63}\right)=\left(\begin{array}{l}
63 \\
n
\end{array}\right)\left[P\left(s_{71}\right)\right]^{n}\left[1-P\left(s_{71}\right)\right]^{63-n}
$$

where $\left(\begin{array}{l}63 \\ n\end{array}\right)$ is the number of combinations of $\mathrm{n}$ events from 63.

\section{Results}

\section{Marker inheritance}

Owing to the lack of polymorphism at one SSR marker (Cyp293), segregations could be analyzed for only six of the seven-microsatellite loci. No significant differences between direct and reciprocal crosses were observed; consequently all the progeny produced by the same parents were pooled to increase the sample size and consequently the statistical power of the segregation tests.

Segregations were tested in the three full-sib families for four loci (Cyp84, Cyp101, Cyp257 andCyp258) and in 2 full-sib families for 2 loci (families Cs129 $\times$ Cs32 and Cs48 $\times$ Cs62 for Cyp52; families Cs79 $\times$ Cs18 and Cs48 $\times$ Cs62 for Cyp174) (allele segregation raw data are provided in supplementary information). The observed progeny profiles were always consistent with those of their parents, except in four cases for which the presence of one null allele at one parent genotype was hypothesized. The Mendelian segregation hypothesis was never rejected. The $P$-values computed from Monte Carlo simulations were highly significant except in two cases (cs79 $\times$ cs18 family for Cyp101 and cs $48 \times$ cs62 family for Cyp258) where probabilities were 8 and $17 \%$, respectively.

SSR profiles of the $C$. sempervirens progeny produced by C. dupreziana seed trees

High allelic diversity was observed among the 63 diploid C. sempervirens seedlings originating from C. dupreziana seed trees. The number of alleles per SSR locus varied from 6 to 8 and within site diversity was always high (Table 1). Frequencies of null and non-null alleles were estimated from progeny genotypes (Figure 1). Only three seedlings exhibited double-band profiles at 2, 4 or 5 loci. The 60 other seedlings had single-band (or no band) profiles and most of them were unique (57 out of 60 ). Three single-band profiles were observed twice (see raw data as supplementary material). The absence of bands at three loci (Cyp101, Cyp257 and Cyp293) for respectively 4 ,

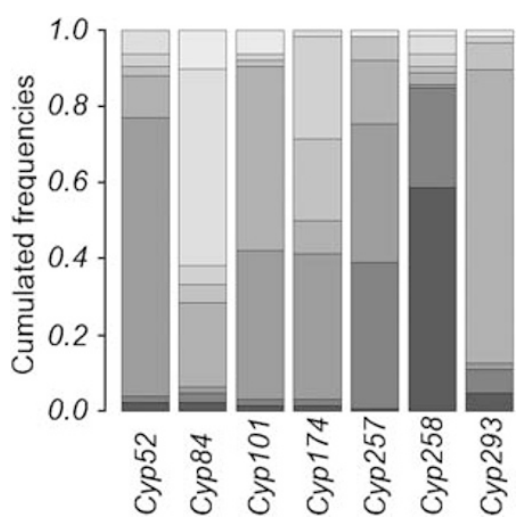

Figure 1 Cumulated single sequence repeat (SSR) allele frequencies estimated in French Cupressus sempervirens populations from the 63 all-paternal progeny produced by Cupressus dupreziana seed trees. Grey scale boxes (from bottom to top): allele frequencies ordered by increasing DNA fragment size. Top boxes of the Cyp101, Cyp257 and Cyp293 markers correspond to null allele. 
1 and 1 seedlings was attributed to the presence of two homologous null alleles (amplification of these samples was repeated three times).

Using allele frequencies estimated for French populations (present study) or for Italian populations (Valgimigli, 2005), the probabilities of observing one single-band genotype over the seven SSR loci in a random mating population were 0.0019 and 0.0035, respectively. Consequently, the probability to observe 60 single-band genotypes among 63 samples is close to zero (1e-159 and $2 \mathrm{e}-143)$. Although there is no evidence for relaxing the hypothesis of linkage equilibrium among loci, some disequilibrium would not strongly affect this computed probability.

The genotypes of the six progeny produced by controlled pollination confirmed the all-paternal origin of these seedlings (see raw data). Indeed progeny produced by parents that do not share alleles exhibited only pollen-tree alleles: a single allele was transmitted to the progeny.

\section{Discussion}

\section{Inheritance of the SSR markers}

The analysis of allele segregations among the 77 C. sempervirens progeny produced by controlled crosses of $C$. sempervirens parents confirmed the putative Mendelian inheritance of the SSR markers developed by Sebastiani et al. (2005).

Mendelian inheritance and high polymorphism make nuclear SSR markers very efficient for the evaluation of heterozygosity (vs homozygosity) in diploids (Chani et al., 2000; Hofer et al., 2002; Fei Yu et al., 2004; Vanwynsberghe et al., 2005). However, null alleles are frequently observed and have to be considered for genetic analysis (Dakin and Avise, 2004; Hoffman and Amos, 2005).

\section{SSR allele diversity}

The high allelic polymorphism observed in the two sets of analyzed progeny (from C. sempervirens controlled crosses and from C. dupreziana seed trees) is in agreement with previous results reported for C. sempervirens populations (Valgimigli, 2005). Genetic diversity in French cypress populations is similar to that in Italian populations. The null allele frequencies estimated in this study for French populations are of the same magnitude as those found by Sebastiani et al. (2005) (from 0.06 to 0.19 for loci Cyp52, Cyp139, Cyp258 and Cyp293).

\section{Ontogenic origin of the diploid all-paternal \\ C. sempervirens seedlings}

The SSR profiles of the six seedlings produced by controlled pollinations unambiguously confirm the allpaternal origin of the seedlings. Indeed alleles that are present in only one of the two parents, either the C. sempervirens pollen tree(s) or the $C$. dupreziana seed tree, demonstrate the absence of a genetic contribution from the seed tree.

According to previous observations, C. sempervirens produces haploid and uninucleated pollen grains (Pichot and El Maâtaoui, 2000). Diploid pollen was not observed in this species. Consequently, the diploid all-paternal C. sempervirens seedlings are likely to derive from the diploidization of one haploid male gamete or from the fusion of two haploid male gametes deriving from one or two pollen grain(s). Indeed, in most conifers each haploid microgametophyte produces several male gametes through mitotic divisions (12 in C. arizonica and 20 in Cupressus goveniana according to Gaussen et al., 1982). The lack of double-band SSR profiles in the progeny originating from heterozygous pollen trees (eight occurrences) suggests a single pollen grain origin of the diploid seedlings. Consequently, the all-paternal C. sempervirens homozygous seedlings are likely to derive from a haploid pollen genotype, either by the diploidization of an initially haploid embryo or by the fusion of two gametes, with identical genotypes, produced by the same pollen grain.

A similar conclusion can be drawn from the 57 progeny produced by the open-pollination of C. dupreziana seed trees. A very high allelic polymorphism was observed within each of the five locations, where seeds were collected and all but three genotypes were unique. These results indicate that numerous pollen trees contributed to the pollen clouds. Most of these progeny (54) exhibited single-band SSR profiles, which is not compatible with a bi-parental origin. The high SSR allele polymorphism observed within each stand (Table 1) means that the likelihood of even one individual with a single-band profile is very low. Computations based on the allele frequencies estimated in Italian cypress population lead to the same conclusion. Moreover, the all-paternal origin of the seedlings allows rejection of the hypothesis of self-pollination that would have increased the probability of observing single-band genotypes. Consequently, we conclude that single-band progeny originate from single-pollen grains.

Excluding the possibility that seed handling or labeling errors occurred, the production of three heterozygous $C$. sempervirens diploids provides evidence for the fusion of two male gametes produced by two different pollen grains. The very low proportion of these heterozygous individuals ( 3 out of 63) suggests that most of the homozygous $C$. sempervirens seedlings originate from the diploidization of a haploid gamete. Therefore, both processes of male-gamete fusion and of male-gamete diploidization must contribute to the production of diploid C. sempervirens progeny from C. dupreziana seed trees.

\section{Use and importance of homozygous lines}

Pure lines are intensively used for selective breeding purposes. They allow for a very efficient estimation of parent-breeding additive value and trait heritability (Gallais, 1990; Bijma et al., 1997). When fertile, they are easily multiplied and produce gametes with an invariable genotype allowing the production of identical offspring such as F1 hybrids. They are also of great interest for genetic mapping, QTL analysis (Picard et al., 1994) and genome sequencing (Komen and Thorgaard, 2007), and facilitate the study of gene expression because recessive alleles cannot be masked by dominant ones as occurs at heterozygous loci ( $\mathrm{Fu}$ and Jansen, 2006).

In the wild, diploid organisms generally originate from the sexual reproduction of two genetically distinct parents and are mostly heterozygous. In normal sexual reproduction, full homozygous individuals can only be 
produced either from homozygous parents by selfing or by cross-pollination within a pure line. For allogamous species, sexual reproduction between genetically related individuals shows deleterious recessive mutations leading to inbreeding depression and natural selection acts to purge this genetic load (Charlesworth and Charlesworth, 1987, 1999; Keller and Waller, 2002). The reduced growth and survival rates observed in the diploid all-paternal $C$. sempervirens progeny as compared with wild C. sempervirens progeny used as controls was attributed to inbreeding depression (Pichot et al., 2008). Inbreeding depression effect is more rapidly observable in diploidization than in selfing because selfing only halves the number of heterozygous loci per generation, whereas diploidization directly produces full homozygous offspring.

In the present study, the good growth performances of the three heterozygous all-paternal C. sempervirens seedlings compared with those of the homozygous diploids are in agreement with this hypothesis, but need to be confirmed with larger sample sizes.

The artificial production of homozygous lines was historically based on several generations of selfing (that only halves the number of heterozygous loci at each generation) or mating between relatives when selfing is not possible. Today it mainly relies on the diploidization of haploid lines produced by gyno- or androgenesis from haploid tissues (Hosp et al., 2007). According to Forster et al. (2007), 50\% of the European barley cultivars are produced by a doubled haploid system. In plants, most haploid lines derive from the in vitro culture of anthers or immature pollen grains. Haplo-diploid individuals are also produced in some animal species such as fishes, using irradiated gametes (Komen and Thorgaard, 2007). This strategy deeply shortens the breeding period especially for species exhibiting long-term cycles. It is frequently used in angiosperms (Forster et al., 2007), including trees (Bueno et al., 2004; Assani et al., 2006; Germana, 2006) but has not succeeded to date in gymnosperms. In this paper, we demonstrate that a new strategy is available for $C$. sempervirens for the production of haploid or diploid homozygous lines by androgenesis in a C. dupreziana surrogate mother. This strategy should improve the efficiency of future breeding programs for the production of ornamental or windbreak C. sempervirens varieties and could be tested in other related species. Our findings also confirm the intrinsic embryogenic ability of pollen grains as frequently reported in angiosperms.

\section{Acknowledgements}

We thank B Jouaud, T Langlois, J Thévenet and A Roig for their contribution to seedling production and SSR genotyping. We also thank B Fady for valuable comments on the paper. This study was partly supported by a PhD grant attributed to JL Rivera Nava by the CONACYT (Consejo Nacional de Ciencia y Tecnologia, Mexico City, Mexico).

\section{References}

Abdoun F, Beddiaf M (2002). Cupressus dupreziana A. Camus: répartition, dépérissement et régénération au Tassili n'Ajjer, Sahara central. C R Biologies 325: 617-627.
Assani A, Gupta B, Rajam MV (2006). Haploid production in trees, ornamental and floricultural plants. In: Teixeira da Silva JA (ed). Floriculture, ornamental and plant biotechnology. p 646.

Barton NH, Charlesworth B (1998). Why sex and recombination? Science 281: 1986-1990.

Bell G (1982). The Masterpiece of Nature: The Evolution and Genetics of Sexuality. University of California Press: Berkeley, California.

Bijma P, Arendonk JAM, Bovenhuis B (1997). Breeding value and variance component estimation from data coming containing inbred individuals: application to gynogenetic families in common carp (Cyprinus carpio L.). Genetics 145: 1243-1249.

Bueno MA, Pintos B, Prado MJ, Gomez A, Manzanera JA (2004). Androgenesis: a tool for woody plant breeding. In: Pandalai SG (ed). Recent Research Developments in Genetics \& Breeding. Vol. 1, Part II, pp 365-383.

Chani E, Veilleux RE, Boluarte-Medina T (2000). Improved androgenesis of interspecific potato and efficiency of SSR markers to identify homozygous regenerants. Plant Cell Tiss Org Cult 60: 101-112.

Charlesworth B, Charlesworth D (1999). The genetic basis of inbreeding depression. Genet Res 74: 329-340.

Charlesworth D, Charlesworth B (1987). Inbreeding depression and its evolutionary consequences. Annu Rev Ecol Syst 18: 237-268.

Dakin EE, Avise JC (2004). Microsatellite null alleles in parentage analysis. Heredity 93: 504-509.

Dugas V (1996). Analyse de la variabilité enzymatique de 5 provenances naturelles et 10 provenances artificielles françaises de cyprès vert (Cupressus sempervirens L.). Rapport de stage de MST 'Chimie et biologie végétales'. Université Claude Bernard-Lyon I.

Fei Yu T, Fei D, GuoYing W (2004). Identification of doubled haploid plants from maize gynogenesis by SSR molecular marker. Acta Agriculturae Universitatis Jiangxiensis 26: 859-862.

Foroughi-Wehr B, Wenzel G (1993). Andro- and parthenogenesis. In: Hayward MD, Bosemark NO, Romagosa I (eds). Plant Breeding: Principles and prospects. Chapman and Hall: London. pp 261-277.

Forster BP, Heberle-Bors E, Kasha KJ, Touraev A (2007). The resurgence of haploids in higher plants. Trends Plant Sci 12: 368-375.

Gallais A (1990). Théorie de la Sélection En Amélioration Des Plantes. Masson: Paris.

$\mathrm{Fu}$ J, Jansen RC (2006). Optimal design and analysis of genetic studies on gene expression. Genetics 172: 1993-1999.

Gaussen H, Leroy JF, Ozenda P (1982). Précis de Botanique, Tome II: Végétaux Supérieurs. Masson: Paris.

Germana M (2006). Doubled haploid production in fruit crops. Plant Cell Tiss Org Cult 86: 131-146.

Hofer M, Gomez A, Aguiriano E, Manzanera JA, Bueno MA (2002). Analysis of simple sequence repeat markers in homozygous lines of apple. Plant Breeding 121: 159-162.

Hoffman JI, Amos W (2005). Microsatellite genotyping errors: detection approaches, common sources and consequences for paternal exclusion. Mol Ecol 14: 599-612.

Hosp J, de Faria Maraschin S, Touraev A, Boutilier K (2007). Functional genomics of microspore embryogenesis. Euphytica 158: 275-285.

Keller LF, Waller DM (2002). Inbreeding effects in wild populations. Trends Ecol Evol 17: 230-241.

Komen J, Thorgaard GH (2007). Androgenesis, gynogenesis and the production of clones in fishes: a review. Aquaculture $\mathbf{2 6 9}$ 150-173.

McKone MJ, Halpern SL (2003). The evolution of androgenesis Am Nat 161: 641-656.

Picard E, Crambes E, Liu CS, Mihamou-Ziyyat A (1994). Evolution of doubled haploid methods and consequences 
for plant breeding. Comptes Rendus des Séances de la Société de Biologie et de ses Filiales 188: 109-141.

Pichot C, Borrut A, El Maâtaoui M (1998). Unexpected DNA content in the endosperm of Cupressus dupreziana A. Camus seeds and its implications in the reproductive process. Sex Plant Reprod 11: 148-152.

Pichot C, El Maâtaoui M (2000). Unreduced diploid nuclei in Cupressus dupreziana A. Camus pollen. Theor Appl Genet 101: 574-579.

Pichot C, El Maâtaoui M, Raddi S, Raddi P (2001). Surrogate mother for endangered Cupressus. Nature 412: 39.

Pichot C, Liens B, Rivera Nava JL, Bachelier JB, El Maâtaoui M (2008). Cypress surrogate mother produces haploid progeny from alien pollen. Genetics 178: 379-383.
R Development Core Team (2006). R: A Language and Environment for Statistical Computing. R Foundation for Statistical Computing: Vienna, Austria.

Sebastiani F, Buonamici A, Fineschi S, Racchi ML, Raddi P, Vendramin GG (2005). Novel polymorphic nuclear microsatellites in Cupressus sempervirens L. Mol Ecol Notes 5: 393-394.

Valgimigli MC (2005). Genetic variability in Italian populations of Cupressus sempervirens L. assessed by SSR and RAPD markers. PhD dissertation, Università degli Studi di Verona, Italy.

Vanwynsberghe L, De Witte K, Coart E, Keulemans J (2005). Limited application of homozygous genotypes in apple breeding. Plant Breeding 124: 399-403.

West SA, Lively CM, Read AF (1999). A pluralistic approach to sex and recombination. J Evol Biol 12: 1003-1012.

Supplementary Information accompanies the paper on Heredity website (http://www.nature.com/hdy) 